\title{
Distinct Cell Transcriptomic Landscapes Upon Henipavirus Infections
}

\author{
Mingyue Chen 1,2,3, Mary Tachedjian², Glenn A. Marsh', Jie Cuii,3* and Lin-Fa Wang4,5* \\ ${ }^{\prime}$ Key Laboratory of Fermentation Engineering, National 111 Center for Cellular Regulation and Molecular Pharmaceutics, \\ Hubei University of Technology, Wuhan, China, ${ }^{2}$ CAS Key Laboratory of Molecular Virology and Immunology, Institut Pasteur \\ of Shanghai, Chinese Academy of Sciences, Shanghai, China, ${ }^{3}$ Center for Biosafety Mega-Science, Wuhan Institute \\ of Virology, Chinese Academy of Sciences, Wuhan, China, ${ }^{4}$ Australian Animal Health Laboratory, CSIRO Health and \\ Biosecurity, Geelong, VIC, Australia, ${ }^{5}$ Programme in Emerging Infectious Diseases, Duke-NUS Medical School, Singapore, \\ Singapore
}

OPEN ACCESS

Edited by:

Ahmed Sayed Abdel-Moneim, Taif University, Saudi Arabia

Reviewed by: Alexander Freiberg, The University of Texas Medical Branch at Galveston, United States

Rekha Khandia,

Barkatullah University, India

*Correspondence: Jie Cui

jcui@ips.ac.cn

Lin-Fa Wang

linfa.wang@duke-nus.edu.sg

Specialty section:

This article was submitted to Virology,

a section of the journal Frontiers in Microbiology

Received: 20 January 2020 Accepted: 23 April 2020

Published: 19 May 2020

Citation:

Chen M, Tachedjian M, Marsh GA, Cui J and Wang L-F (2020) Distinct Cell Transcriptomic

Landscapes Upon Henipavirus Infections. Front. Microbiol. 11:986.

doi: 10.3389/fmicb.2020.00986
Hendra virus (HeV) and Cedar virus (CedV) are henipaviruses, which fall into the Paramyxoviridae family of single-stranded, negative-sense RNA viruses. HeV is classified as a Biosafety Level-4 (BSL-4) agent, as it is highly pathogenic and is often fatal to humans. To date, no HeV prevention or treatment methods for human are available. In contrast, previous experimental infection studies have suggested that CedV is non-pathogenic. Flying foxes (pteropid bats) in Australia are the natural reservoirs of both viruses, but the cellular responses of bats to these viral infections remain unclear. Here, we infected bat and human cells with these viruses. We then examined the total transcriptomic landscapes of the cells at 6 or $24 \mathrm{~h}$ post infection. Unexpectedly, despite the close phylogenetic relationship between $\mathrm{HeV}$ and $\mathrm{CedV}$, there was a dramatic difference in cellular gene expression patterns in response to the two different infections. It is likely that minor differences in the phosphoprotein $(P)$ gene coding strategy between the two viruses cause the observed incongruence in host transcriptomic divergence and viral lethality. This study greatly expands our understanding of the pathogenic mechanisms of henipaviruses.

Keywords: bats (Chiroptera), Hendra virus (HeV), Cedar virus, transcriptomatics, cell infection

\section{INTRODUCTION}

Two viruses in the genus Henipavirus (Eaton et al., 2006; Marsh et al., 2012), Hendra (HeV) and Nipah (NiV), are the only known Biosafety Level 4 (BSL-4) pathogens in the family Paramyxoviridae (Marsh et al., 2012). Both of these viruses cause $40-100 \%$ mortality in humans and other animals (Eaton et al., 2006). The natural reservoir of $\mathrm{HeV}$ is the Australian flying foxes (pteropid bats) (Field et al., 2001). Another henipaviral species, Cedar virus (CedV), was isolated from fruit bats in Australia (Marsh et al., 2012). $\mathrm{HeV}$ has a broad host range as it uses a highly conserved cellular protein, ephrin-B2, as an entry receptor (Marsh et al., 2012; Field, 2016). CedV also uses ephrin-B2 (Marsh et al., 2012). However, CedV and HeV use different coding strategy of the viral phosphoprotein $(\mathrm{P})$ gene, which play fundamental role in antagonizing the innate immune system of the host (Marsh et al., 2012; Glennon et al., 2015). Unlike HeV, P gene of CedV lacks the coding capacity for the highly conserved $\mathrm{V}$ and $\mathrm{W}$ proteins (Figure 1A). V protein could be the 


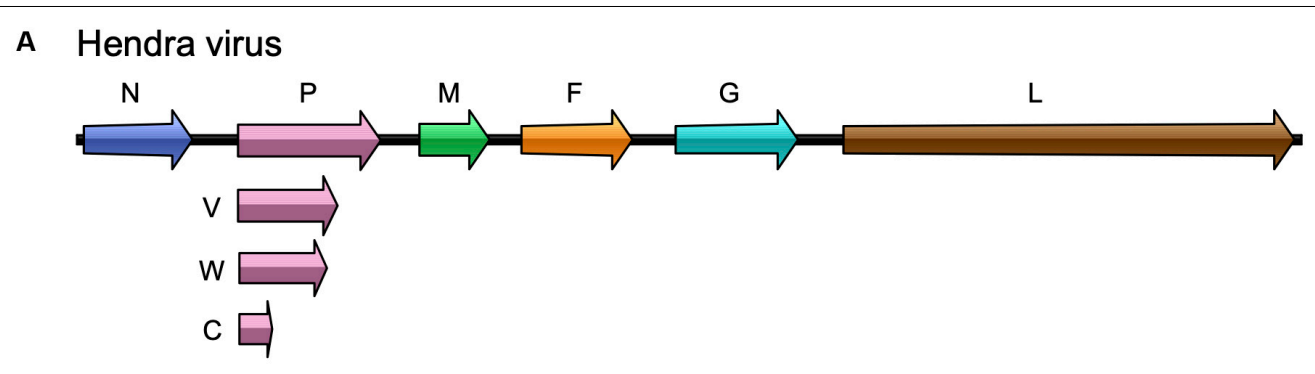

Cedar virus
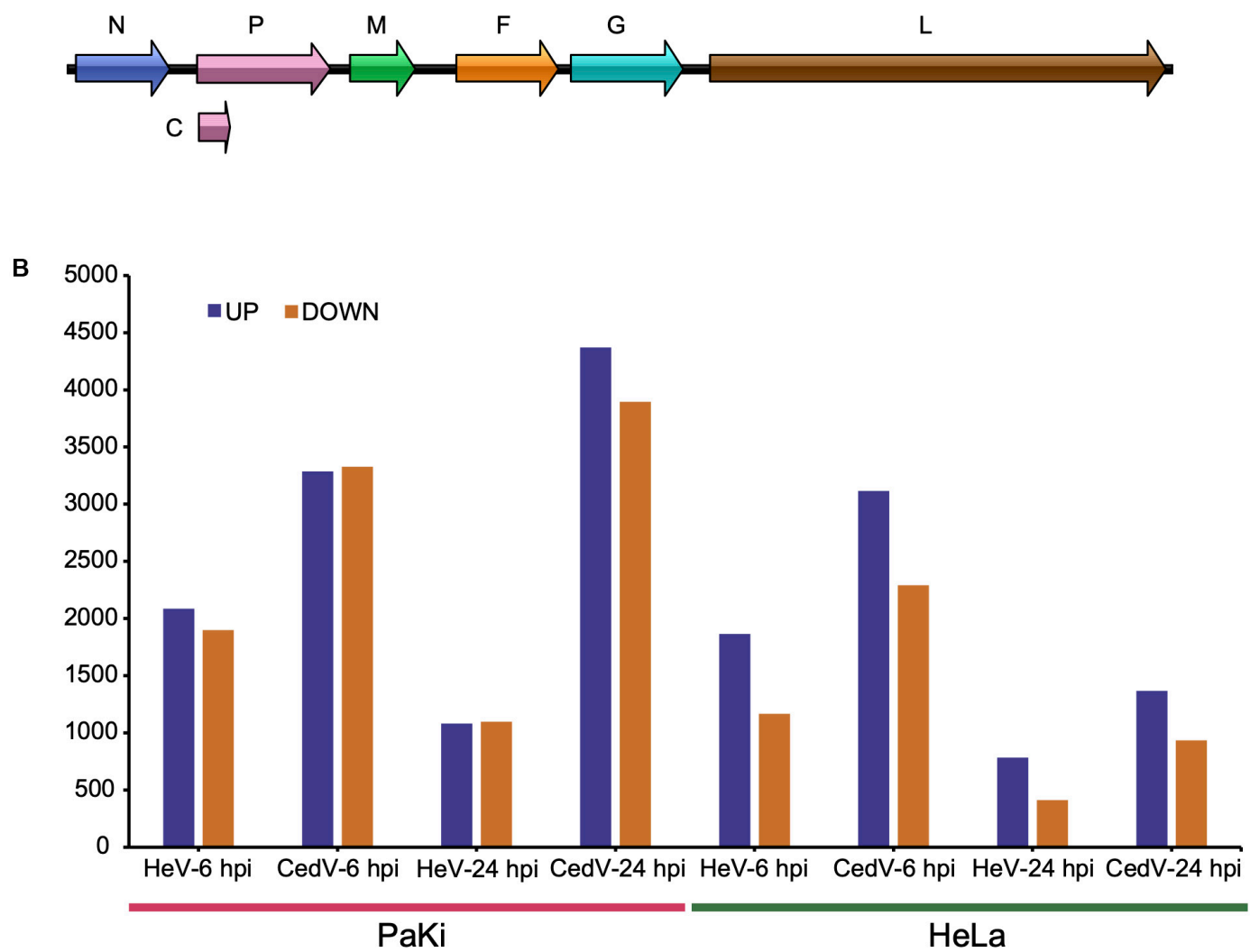

FIGURE 1 | HeV and CedV genome organization and RNA-seq profiles. (A) Genome organization of HeV and CedV. (B) DEGs in HeV- or CedV-infected HeLa and PaKi cells at 6 and 24 hpi. HeV-6 hpi and CedV-6 hpi represent HeV- or CedV-infected corresponding PaKi or HeLa at 6 hpi. HeV-24 hpi and CedV-24 hpi represent HeV- or CedV-infected corresponding PaKi or HeLa at 24 hpi.

major determinant of pathogenesis while $\mathrm{W}$ protein determines the disease course (Satterfield et al., 2015). When ferrets and guinea pigs were experimentally challenged with CedV, viral replication and the generation of neutralizing antibodies were observed, but no clinical disease was identified (Marsh et al., 2012).

Many bat-borne zoonotic viruses are highly pathogenic to spillover hosts, but bats are predominantly clinically asymptomatic and rarely display any signs of disease (Wynne et al., 2014). The mechanisms used by bats to balance the support and control of viral infections remain largely unknown. Transcriptomic profiling of viral infections is ideal for studying these mechanisms. We thus used RNA sequencing (RNA-seq) to identify differences in human and bat cell lines after two periods of infection with either $\mathrm{HeV}$ or CedV. Surprisingly, both cell lines exhibited a stronger immune response to $\mathrm{CedV}$ than to $\mathrm{HeV}$. Our study provides novel insight into the host response to infection with phylogenetically similar, but pathogenically dissimilar viruses. 


\section{RESULTS}

\section{Host Gene Transcription Following Infection With HeV or CedV}

To explore the effects of $\mathrm{HeV}$ and $\mathrm{CedV}$ on host gene expression, we used RNA-seq to analyze $\mathrm{HeV}$-, CedV-, or mock-infected immortalized cells lines from bats (PaKi) and humans (HeLa) 6 and $24 \mathrm{~h}$ post infection (hpi). Across all infection groups, the most differentially expressed genes (DEGs) were identified in CedVinfected PaKi cells at 24 hpi (4371 genes upregulated and 3896 downregulated, as compared to uninfected PaKi cells; Figure 1B). The fewest DEGs were identified in HeV-infected HeLa cells at 24 hpi (784 genes upregulated and 411 downregulated, as compared to uninfected HeLa cells). And more DEGs were identified in the CedV-infected cells than in $\mathrm{HeV}$-infected cells, irrespective of cell type. Therefore, the host response to $\mathrm{HeV}$ and $\mathrm{CedV}$ differed, with CedV inducing more differential expression of host genes than $\mathrm{HeV}$. In addition, more DEGs were identified in the bat cells than in the human cells, irrespective of infection type.

\section{Pattern Recognition Receptors (PRRs)}

PRRs, which include toll-like receptors (TLRs) and retinoic acid inducible gene I (RIG-I) like receptors (RLRs), are vital to the host immune system as they form the first line of defense against infection (Baker et al., 2013). The RLR family comprises RIG-I, melanoma differentiation-associated protein 5 (MDA5), laboratory of genetics and physiology 2 (LGP2) (Satoh et al., 2010). RIG-I, MDA5, LGP2, and TLR3 were significantly upregulated in CedV-infected PaKi and HeLa cells at $24 \mathrm{hpi}$ (Table 1). These genes were more strongly upregulated in PaKi cells than in HeLa cells.

NOD-like receptor family CARD domain containing 5 (NLRC5) is an important regulator of MHC class I gene expression (Ranjan et al., 2015). NLRC5 interacts with RIG-I to induce a robust response to the influenza virus; overexpression of NLRC5 resulted in impaired influenza viral replication (Ranjan et al., 2015). Here, NLRC5 was upregulated in CedV-infected $\mathrm{PaKi}$ and HeLa cells at $24 \mathrm{hpi}$, with more differential expression observed in PaKi cells. This indicated that NLRC5 may also interact with RIG-I to impair CedV replication. The presence of highly expressed PRRs in both CedV-infected cell lines suggested that both bats and humans have a strong antiviral response to CedV infection.

\section{Interferon (IFN) Response to Infection}

To determine the role of IFNs in the host response to viral infections, RNA-seq reads from PaKi cells (HeV-, CedV-, or mock-infected) were separately mapped to type I IFN locus (three IFN- $\alpha$, one IFN- $\beta$, one IFN- $\varepsilon$, and five IFN- $\omega$ ), using the type I IFN locus from Pteropus alecto as a reference (Zhou et al., 2016). IFN- $\alpha 1$, IFN- $\alpha 2$, IFN- $\alpha 3$, IFN- $\beta$, IFN- $\omega 3$, IFN- $\omega 4$, and IFN- $\omega 5$ were highly expressed in CedV-infected PaKi cells at 24 hpi, based on the read depth counts of the IFN transcripts, especially IFN$\alpha 2$, IFN- $\alpha 3$, IFN- $\beta$, and IFN- $\omega 4$ (Figure 2). In contrast, type I IFN expression in PaKi cells showed little change following $\mathrm{HeV}$ infection. RNA-seq reads from PaKi cells were also mapped to the IFN- $\lambda 1$ and IFN- $\lambda 2$ genes of P. Alecto (Zhou et al., 2011). At 24 hpi with CedV, IFN- $\lambda 2$ expression in PaKi cells was high, while IFN $-\lambda 1$ expression was relatively low. Consistent with our results for type I IFN genes, IFN- $\lambda 1$ and IFN- $\lambda 2$ expression did not increase in $\mathrm{HeV}$-infected PaKi cells. HeV-, CedV-, or Mockinfected HeLa cells were also mapped against human genome. In CedV-infected HeLa cells, IFN- $\lambda(\lambda 1, \lambda 2$, and $\lambda 3$ ) and IFN$\beta$ were both upregulated at $24 \mathrm{hpi}$, but no IFN genes were differentially expressed in $\mathrm{HeV}$-infected HeLa cells (Table $\mathbf{1}$ ).

IFNs stimulate antiviral activity by inducing interferonstimulated genes (ISGs), including the IFN-induced protein with the tetratricopeptide repeats (IFIT) (Fensterl and Sen, 2015). We examined the expression of IFITs during $\mathrm{HeV}$ and CedV infection. IFIT1, IFIT2, IFIT3, and IFIT5 were significantly upregulated in CedV-infected HeLa and PaKi cells (Table 2). Indeed, the expression of IFIT1, IFIT2, and IFIT3 increased over 2000-fold in the CedV-infected PaKi cells, as compared to uninfected PaKi cells. Unexpectedly, IFIT1, IFIT2, and IFIT3 were also upregulated in $\mathrm{HeV}$-infected HeLa cells at 24 hpi.

The $2^{\prime}, 5^{\prime}$-oligoadenylate synthetase (OAS) proteins can be induced by type I IFNs (Sadler and Williams, 2008). Consistent with this, OAS2, OAS3, and OASL were significantly upregulated in CedV-infected HeLa and PaKi cells at $24 \mathrm{hpi}$, with OAS1 upregulated in CedV-infected PaKi cells at 24 hpi. The HeV V and $\mathrm{W}$ proteins block IFN production, with $\mathrm{V}$ inhibiting MDA5

TABLE 1 | Log2 fold change (log2FC) of DEGs involved in pattern recognition in HeV- or CedV-infected PaKi and HeLa cells.

\begin{tabular}{|c|c|c|c|c|c|c|c|c|}
\hline & \multicolumn{4}{|c|}{ PaKi } & \multicolumn{4}{|c|}{ HeLa } \\
\hline MDA5 & -0.49 & -0.84 & 0.52 & 7.51 & - & - & 1.79 & 6.36 \\
\hline LGP2 & - & - & - & 9.68 & - & - & - & 3.99 \\
\hline TLR3 & -0.95 & -0.42 & 0.59 & 2.14 & - & - & 0.57 & 3.02 \\
\hline NLRC5 & - & - & - & 9.72 & - & -0.59 & - & 0.75 \\
\hline
\end{tabular}

${ }^{a} \mathrm{HeV}$-infected corresponding PaKi or HeLa cell at 6 hpi. ${ }^{b} \mathrm{CedV}$-infected corresponding PaKi or HeLa cell at 6 hpi. ${ }^{c} \mathrm{HeV}$-infected corresponding PaKi or HeLa cell at 24 hpi. ${ }^{d} \mathrm{CedV}$-infected corresponding PaKi or HeLa cell at $24 \mathrm{hpi}$. ${ }^{e}$ The corresponding gene was not differentially expressed. 


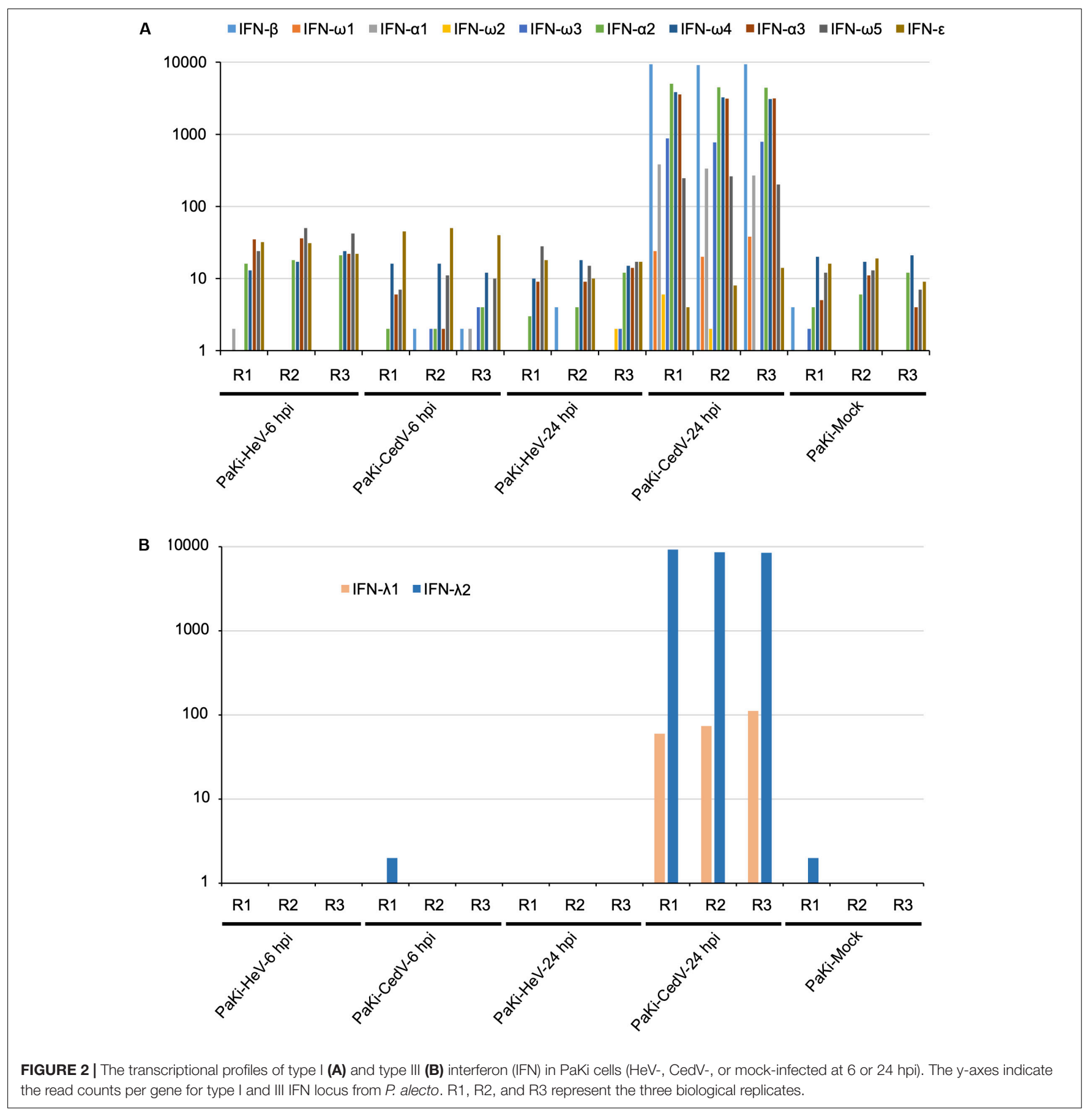

signaling and $\mathrm{W}$ blocking IFN synthesis from TLRs after IFN regulatory factor 3 (IRF3) activation (Glennon et al., 2015). At 24 hpi, MDA5 and TLRs were upregulated in CedV-infected PaKi and HeLa cells, while IRF3 was upregulated in CedV-infected PaKi cells only (Table 2).

\section{Apoptosis}

Several host mechanisms that prevent viral replication, viral dissemination, or persistent viral infection involve programmed cell death, or apoptosis (Barber, 2001). The extrinsic apoptosis pathway is triggered by the binding of death receptors (TNF receptor 1 (TNFR1), TNF-related apoptosis-inducing ligand (TRAIL) receptor 1/2 (TNFRSF10A/B), and FasL-related receptor Fas to their respective ligands (TNF- $\alpha$, TRAIL, and FasL) (Cuda et al., 2016). At 24 hpi, the ligands TNF$\alpha$ and TRAIL, death receptors TNFR1, TNFRSF10B, Fas, as well as apoptosis signaling protein DAXX were upregulated in CedV-infected PaKi cells, suggesting that TNF- $\alpha-$, TRAIL-, and FasL-mediated apoptosis may be induced (Figure 3). And the expression of TNF- $\alpha$, TRAIL, TNFRSF10B, and DAXX in 
TABLE 2 | Log2FC of the DEGs involved in the IFN response to HeV and CedV infection in PaKi and HeLa cells.

\begin{tabular}{|c|c|c|c|c|c|c|c|c|}
\hline & \multicolumn{4}{|c|}{ PaKi } & \multicolumn{4}{|c|}{ HeLa } \\
\hline & HeV-6 hpi ${ }^{a}$ & CedV-6 hpi ${ }^{b}$ & HeV-24 hpic & CedV-24 hpi ${ }^{d}$ & HeV-6 hpi & CedV-6 hpi & HeV-24 hpi & CedV-24 hpi \\
\hline IFN- $\beta$ & $N^{e}$ & $\mathrm{~N}$ & $\mathrm{~N}$ & $\mathrm{~N}$ & $--^{f}$ & - & - & 13.92 \\
\hline IFN- $\lambda 1$ & $N$ & $\mathrm{~N}$ & $N$ & $\mathrm{~N}$ & - & - & - & 13.36 \\
\hline $\mathrm{IFN}-\lambda 2$ & $\mathrm{~N}$ & $\mathrm{~N}$ & $\mathrm{~N}$ & $N$ & - & - & - & 10.68 \\
\hline IFN- $\lambda 3$ & $\mathrm{~N}$ & $\mathrm{~N}$ & $N$ & $N$ & - & - & - & 10.88 \\
\hline IFIT1 & - & - & - & 11.90 & -0.85 & -1.50 & 1.06 & 5.91 \\
\hline IFIT2 & - & - & - & 11.96 & - & - & 1.46 & 6.53 \\
\hline IFIT3 & - & - & - & 11.00 & 0.36 & 0.60 & 1.23 & 5.86 \\
\hline IFIT5 & - & -0.81 & - & 1.86 & - & 0.51 & - & 2.85 \\
\hline OAS1 & - & - & - & - & - & - & - & 3.71 \\
\hline OAS2 & - & - & - & 8.46 & - & - & - & 9.31 \\
\hline OAS3 & - & -0.62 & 0.52 & 5.85 & 0.54 & 0.24 & 0.53 & 3.66 \\
\hline OASL & - & - & - & 11.42 & - & - & 1.18 & 5.22 \\
\hline IRF3 & - & - & - & 0.93 & - & - & - & - \\
\hline
\end{tabular}

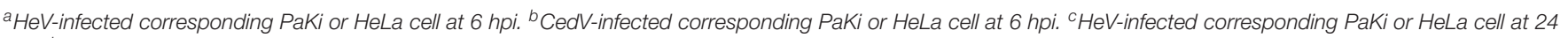

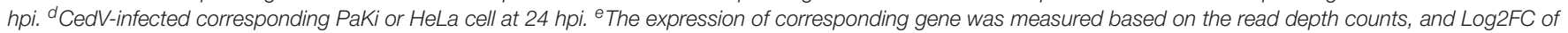
was not calculated. ${ }^{f}$ The corresponding gene was not differentially expressed.

CedV-infected PaKi increased more than 3-fold over uninfected PaKi cells (Table 3). According to Table 3, bat induced a relatively stronger apoptosis response at $24 \mathrm{hpi}$ than at 6 hpi. However, at $24 \mathrm{hpi}$, in CedV-infected HeLa cells, TNFR1, TNFRSF10A, TNFRSF10B and Fas were only slightly upregulated as compared to uninfected HeLa cells. In addition, few genes were upregulated in $\mathrm{HeV}$-infected PaKi and $\mathrm{HeLa}$ cells at 6 and 24 hpi.

Caspase- 8 was only upregulated in CedV-infected PaKi and HeLa cells, as well as in $\mathrm{HeV}$-infected PaKi cells at $24 \mathrm{hpi}$. There was an over 2-fold increase in the expression of caspase8 in CedV-infected PaKi cells, as compared to uninfected cells. It is therefore probable that CedV sensitizes human and bat cells to TNF- $\alpha$-, TRAIL-, and FasL-mediated apoptosis more effectively than does $\mathrm{HeV}$ (Figure 3). The overexpression of caspase-7 indicated that this protein may be also involved in apoptosis-mediated cell death. Caspase-3 was only upregulated in HeV-infected HeLa cells (Table 3). At 24 hpi, another proapoptotic group of genes (DAXX, RIP1, TRAF2, ASK1, JUN, AP1, and Bim) were induced by over 2-fold in CedV-infected $\mathrm{PaKi}$ cells, implying that PaKi cells were also subjected to strong pro-apoptotic signals.

\section{Cytokines}

In response to viral infection, target cells produce cytokines and chemokines to control viral replication (Klotman and Chang, 2006). Here, the cytokines IL6, IL8, CCL2, CXCL2, and CXCL16 were upregulated in $\mathrm{HeV}$ - or CedV-infected PaKi and HeLa cells at 6 and $24 \mathrm{hpi}$, suggesting that cytokines may function during $\mathrm{HeV}$ or $\mathrm{CedV}$ infection in PaKi and HeLa cells. It is notable that more cytokines were significantly upregulated in CedV-infected PaKi cells than in $\mathrm{HeV}$-infected PaKi cells or in HeLa cells infected with either virus at 24 hpi (Figure 4). In particular, the expression levels of TNF- $\alpha$, IL12A, CCL5, and CCL8 increased over 1000-fold in CedV-infected PaKi cells, as compared to uninfected cells. The large number of highly expressed cytokines in CedV-infected PaKi cells suggested that the bat immune system responds strongly to CedV infection.

\section{NF-кB Signaling}

The nuclear factor- $\mathrm{B}(\mathrm{NF}-\kappa \mathrm{B})$ family of transcription factors regulate innate and acquired host immune responses, and is thus critical for the host response to microbial pathogen infection (Rahman and McFadden, 2011). The mammalian NF- $\kappa$ B proteins are members of the Rel domain-containing protein family: RELA, RELB, c-REL, the NF- $\kappa$ B p105 subunit (NF- $\kappa$ B1), and the NF- $\kappa$ B p100 subunit (NF- $\kappa$ B2). The expression levels of RELA, RELB, c-REL, NF- $\kappa$ B1, NF- $\kappa$ B2, and NF- $\kappa$ B inhibitor- $\alpha$ (I $\kappa \mathrm{B} \alpha)$ were affected by $\mathrm{HeV}$ and $\mathrm{CedV}$ infection in both PaKi and HeLa cells at 6 and $24 \mathrm{hpi}$, but the majority of these genes were most highly expressed in CedV-infected PaKi cells at 24 hpi (Supplementary Table S1). Both TRAF2 and RIP1, which interact with TNFR1 in the classical NF- $\mathrm{B}$ pathway, were highly expressed in CedVinfected PaKi cells at 24 hpi (Table 3), suggesting that TNF- $\alpha$ is involved in the activation of $\mathrm{NF}-\kappa \mathrm{B}$, leading to the transcription of genes that encode pro-inflammatory and proliferative factors. Thus, both $\mathrm{HeV}$ and CedV might induce an immune response in $\mathrm{PaKi}$ and $\mathrm{HeLa}$ cells via NF- $\mathrm{B}$ signaling. The immune response was strongest in CedV-infected PaKi cells.

\section{JAK-STAT Signaling}

The cytokine-activated Janus kinase (JAK)-signal transducer and activator of transcription (STAT) pathway play an important role in the control of immune responses (Shuai and Liu, 2003). Here, JAK2, JAK3, STAT1, STAT2, and STAT3 were over 2-fold upregulated in CedV-infected PaKi cells at 24 hpi. In contrast, at 24 hpi, JAKs and STATs were expressed at relatively low levels in CedV-infected HeLa cells. The expression levels of JAKs and STATs in HeLa and PaKi cells showed little change following $\mathrm{HeV}$ infection (Supplementary Table S2) at 6 and $24 \mathrm{hpi}$, 


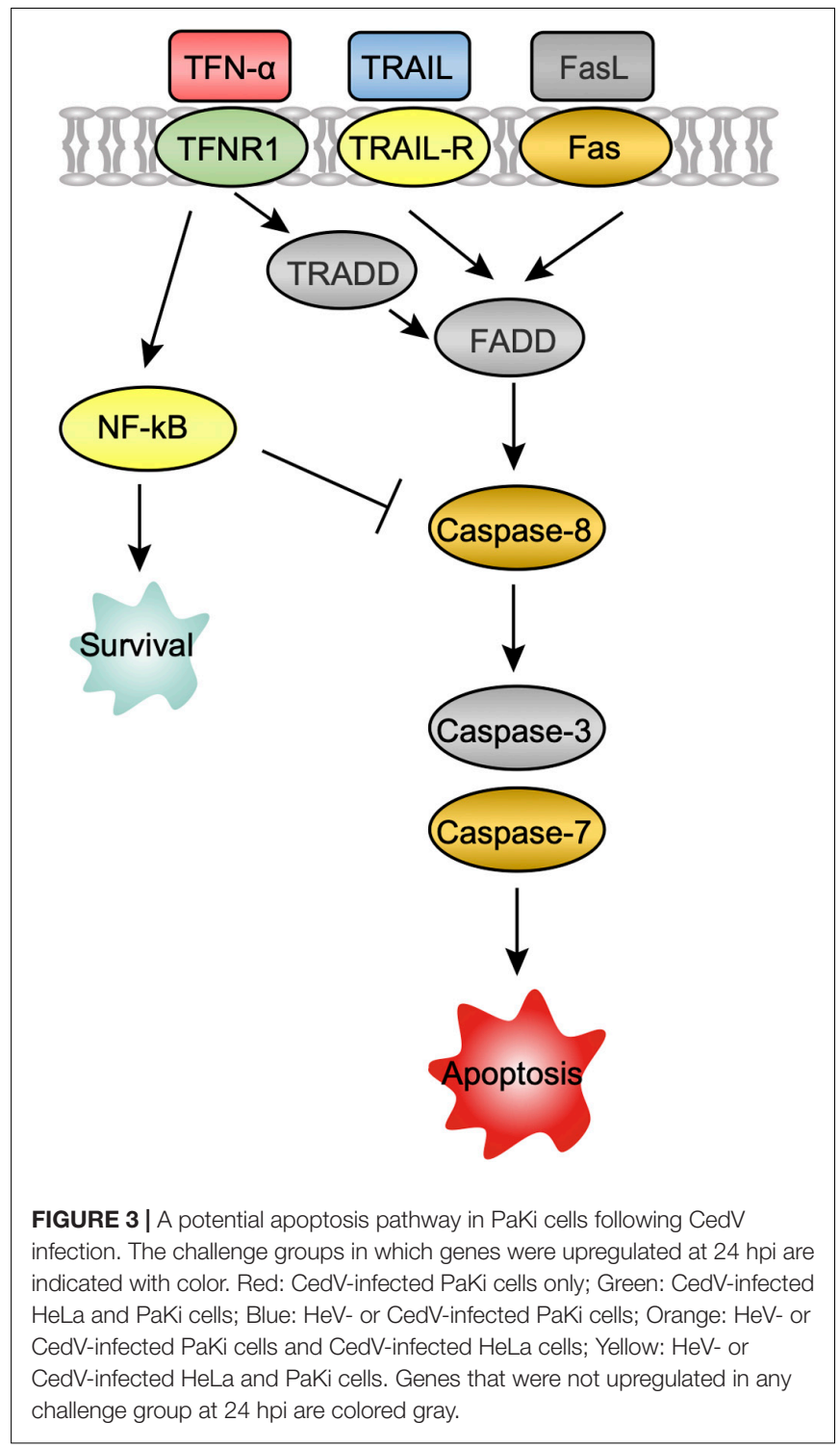

indicating that CedV infection stimulated JAK-STAT signaling but $\mathrm{HeV}$ infection might not. Interestingly, the suppressor of cytokine signaling (SOCS) proteins that regulate the JAKSTAT were also upregulated in CedV-infected PaKi cells at 24 hpi (Supplementary Table S2). As SOCS inhibits JAK-STAT signaling (Shuai and Liu, 2003), its upregulation indicates a classic negative-feedback loop.

\section{Genes Highly Differentially Expressed Following HeV and CedV Infection}

To better understand the functions of genes strongly up- and downregulated post infection, we selected DEGs with $>2$-fold changes in expression level in both cell lines after $\mathrm{HeV}$ or $\mathrm{CedV}$ infection, as compared to uninfected cells. At $24 \mathrm{hpi}$, CedVinfected PaKi cells had the most DEGs with a $>2$-fold change in expression (1441 DEGs upregulated and 1039 downregulated) (Figure 5). At $24 \mathrm{hpi}$, the fewest DEGs with a $>2$-fold change in expression were observed in $\mathrm{HeV}$-infected HeLa cells (46 upregulated and 4 downregulated).

We then used Kyoto encyclopedia of genes and genomes (KEGG) pathway enrichment analysis to predict potential interactions among these selected DEGs. Our KEGG pathway analysis indicated that for DEGs with a $>2$-fold upregulation the most frequently predicted pathways were the TNF signaling pathway, the NF- $\mathrm{B}$ signaling pathway, and the NOD-like receptor signaling pathway (Supplementary Table S3). Notably, all genes significantly upregulated at 6 or 24 hpi in all challenge groups were enriched in the TNF signaling pathway. In addition, selected DEGs upregulated in the HeV-infected PaKi cells, HeVinfected HeLa cells, and CedV-infected HeLa cells were enriched in the NF-кB signaling pathway at $24 \mathrm{hpi}$. However, CedVinfected PaKi cells were not highly enriched in the NF- $\kappa \mathrm{B}$ pathway at $24 \mathrm{hpi}$. We did not perform a KEGG pathway analysis on the selected DEGs downregulated in the HeV-infected HeLa cells at $24 \mathrm{hpi}$, as only four genes were highly downregulated. In contrast to the upregulated genes, distinct pathways were enriched in the downregulated DEGs, and there were no shared pathways across the challenge groups (Supplementary Table S4).

\section{DISCUSSION}

Here, we used the expression profiles of PaKi and HeLa cells infected with $\mathrm{HeV}$ or CedV to identify differences in host response to these henipaviruses. We found that host responses to $\mathrm{HeV}$ and $\mathrm{CedV}$ infection differed dramatically. The large number of DEGs in CedV-infected PaKi cells at 24 hpi indicated that the bat response to $\mathrm{CedV}$ infection was strong. In contrast, relatively few DEGs were identified in $\mathrm{HeV}$-infected HeLa cells at 24 hpi. Various immune related proteins (e.g., PRRs, IFNs, and cytokines) and pathways (e.g., apoptosis, NF- $\kappa \mathrm{B}$ signaling, and JAK-STAT signaling) were upregulated in PaKi and HeLa cells following CedV infection. The immune response was relatively stronger in $\mathrm{PaKi}$ cells than in $\mathrm{HeLa}$ cells. One possible explanation for this discrepancy is that the CedV P gene does not encode $\mathrm{V}$ and $\mathrm{W}$ proteins; these proteins may antagonize host innate immunity, including blocking IFN production (Glennon et al., 2015). Because our results indicated that CedV infection induces interferon expression and other immune signaling pathways in both HeLa and PaKi cells, it is probable that $\mathrm{V}$ and $\mathrm{W}$ proteins affect several pathways related to immunity.

Previous study showed that no clinical disease was observed when Cedar virus was tested in experimental challenge models in ferrets and guinea pigs (Marsh et al., 2012). One explanation for this is that ferrets and guinea pigs may control viral replication as part of an innate antiviral response, such as PRRs, IFNs, cytokines, and apoptosis-related factors. The immune responses of $\mathrm{HeLa}$ and $\mathrm{PaKi}$ cells to $\mathrm{HeV}$ infection were weak, possibly because $\mathrm{V}$ and $\mathrm{W}$ proteins were present. This is consistent with a recent study showing that $\mathrm{V}$ and $\mathrm{W}$ proteins were crucial for pathogenesis and disease progression, respectively (Satterfield et al., 2015).

The greater production of cytokines by CedV-infected PaKi cells compared to $\mathrm{HeV}$-infected PaKi cells observed here was 
TABLE 3 | Log2FC of DEGs involved in apoptosis in HeV- or CedV-infected PaKi and HeLa cells.

\begin{tabular}{|c|c|c|c|c|c|c|c|c|}
\hline & \multicolumn{4}{|c|}{ PaKi } & \multicolumn{4}{|c|}{ HeLa } \\
\hline & HeV-6 hpia & CedV-6 hpi ${ }^{b}$ & $\mathrm{HeV}-24 \mathrm{hpi}^{\mathrm{c}}$ & CedV-24 hpid & HeV-6 hpi & CedV-6 hpi & HeV-24 hpi & CedV-24 hpi \\
\hline TNF- $\alpha$ & $-^{e}$ & - & - & 12.41 & - & - & - & - \\
\hline TRAIL & -1.25 & -3.28 & 0.86 & 3.47 & - & - & - & - \\
\hline TNFR1 & - & 0.84 & - & 0.54 & - & - & - & 0.38 \\
\hline TNFRSF10A & - & - & - & - & 0.74 & 1.01 & - & 0.37 \\
\hline TNFRSF10B & 1.66 & 2.52 & 0.93 & 3.31 & 0.45 & 0.78 & 0.29 & 0.32 \\
\hline FAS & - & 0.24 & 0.44 & 0.95 & - & 0.54 & - & 0.76 \\
\hline FADD & - & - & - & -0.30 & - & - & - & - \\
\hline DAXX & 0.45 & 0.45 & - & 2.29 & - & - & - & - \\
\hline TRADD & - & - & - & - & - & -0.56 & - & - \\
\hline CASP8 & - & - & 0.45 & 1.70 & - & - & - & 0.62 \\
\hline CASP3 & - & -0.23 & - & -1.04 & 0.31 & 0.43 & - & - \\
\hline CASP7 & 0.93 & -0.82 & 0.31 & 0.64 & 0.45 & 0.63 & - & 0.93 \\
\hline RIP1 & - & - & - & 1.11 & - & 0.28 & - & 0.33 \\
\hline TRAF2 & 0.49 & 0.65 & - & 1.36 & 0.61 & 0.71 & - & - \\
\hline AIP & -0.37 & -0.53 & - & -0.99 & - & 0.37 & - & - \\
\hline ASK1 & - & -0.62 & - & 1.47 & 0.29 & - & - & - \\
\hline JNK1 & - & 0.95 & - & 0.27 & - & 0.39 & - & - \\
\hline JNK2 & - & -0.44 & - & -0.82 & - & - & - & - \\
\hline JUN & - & -0.34 & - & 2.11 & 0.62 & 0.88 & - & 0.53 \\
\hline AP1 & - & 1.23 & - & 4.01 & -0.76 & 0.38 & 0.67 & 0.68 \\
\hline p53 & 0.37 & - & 0.42 & 0.59 & -0.95 & -0.95 & 0.32 & 0.40 \\
\hline Bim & - & 1.47 & - & 1.34 & - & 0.77 & - & 0.27 \\
\hline
\end{tabular}

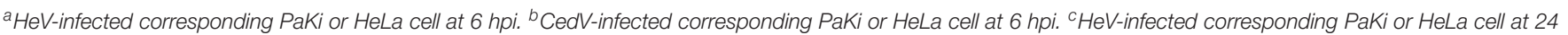
hpi. ${ }^{d} \mathrm{CedV}$-infected corresponding PaKi or HeLa cell at $24 \mathrm{hpi}$. ${ }^{e}$ The corresponding gene was not differentially expressed.

likely due to the absence of the $\mathrm{W}$ protein in $\mathrm{CedV}$, as it has been shown that cells infected with $\mathrm{W}$-deficient $\mathrm{NiV}$ produce more cytokines than in cells infected with wild-type NiV (Satterfield et al., 2015). The $\mathrm{W}$ protein may control the cytokine response of target endothelial cells, thus affecting disease progression without altering disease lethality (Satterfield et al., 2015). Therefore, although cytokine production may not be directly responsible for the distinct clinical outcomes of $\mathrm{HeV}$ and $\mathrm{CedV}$ infections, cytokine production may affect disease progression.

Several genes upregulated in $\mathrm{HeV}$-infected $\mathrm{HeLa}$ and $\mathrm{PaKi}$ cells encoded cytokines or elements of the NF- $\mathrm{B}$ signaling pathway. Moreover, our KEGG pathway analysis of highly upregulated DEGs indicated that the TNF and NF-кB signaling pathways were enriched in $\mathrm{HeV}$-infected HeLa and PaKi cells, suggesting that $\mathrm{HeV}$-infection induced certain innate antiviral responses in both cell lines. Several proteins crucial to apoptosis, such as TRAIL, TNFRSF10B, FAS, CASP7, and CASP8, were upregulated in $\mathrm{HeV}$-infected $\mathrm{PaKi}$ cells at $24 \mathrm{hpi}$, suggesting that $\mathrm{HeV}$ infection may trigger apoptosis in PaKi cells. This is consistent with a previous study, which indicated that $\mathrm{HeV}$ induces apoptosis in bat cells, but not human cells (Wynne et al., 2014). Both $\mathrm{HeV}$ and CedV could induce apoptosis in bat, which implied that apoptosis pathway may contribute to control the viral infection in bat. However, apoptosis could not be induced by $\mathrm{HeV}$ in HeLa cells and the immune response is extremely weak compared to HeLa cells infected with CedV, thus may result in divergent clinical outcomes of human infected with $\mathrm{HeV}$ or CedV. A comparative analysis based on the relative codon deoptimization index (RCDI) for host adaptation of $\mathrm{HeV}$, CedV, Nipah virus, and Hendra like Mojiang virus revealed that except for dog and hamster, all other evaluated hosts (human, bat, horse, pig, cat, ferret, squirrel monkey, and African green monkey) were most susceptible to $\mathrm{HeV}$ while all hosts were least susceptible to CedV (Khandia et al., 2019), which may also provide insight to the distinct clinical outcomes of $\mathrm{HeV}$ and CedV. At $24 \mathrm{hpi}$, the expression levels of IFIT1, IFIT2, and IFIT3 were over 2fold up-regulated in $\mathrm{HeV}$-infected HeLa cells (Table 2), probably because genes such as IRF7 can induce the expression of ISGs in the absence of type I or III IFN (Schmid et al., 2010). In PaKi cells, there were several DEGs (e.g., MDA5, TLR3, IFIT5, TRAIL, and JUN) downregulated at 6 hpi but significantly upregulated at $24 \mathrm{hpi}$, especially infected with CedV. In general, the host response to viral infection is stronger at $24 \mathrm{hpi}$ than that at $6 \mathrm{hpi}$. Probably $24 \mathrm{~h}$ post infection could be more appropriate to analyze the host response.

We observed several points of similarity between the two viruses. For example, some DNA damage checkpoint and innate immune genes shown to be under positive selection in bats (e.g., p53, c-REL, and RAD50) (Zhang et al., 2013) were upregulated in $\mathrm{PaKi}$ cells infected with either virus, suggesting that the certain innate antiviral mechanisms activated by henipaviruses are conserved across bat lineages. In summary, we used RNA-seq to compare bat and human cell lines infected with $\mathrm{HeV}$ or CedV. We found that CedV caused a stronger innate immune response 

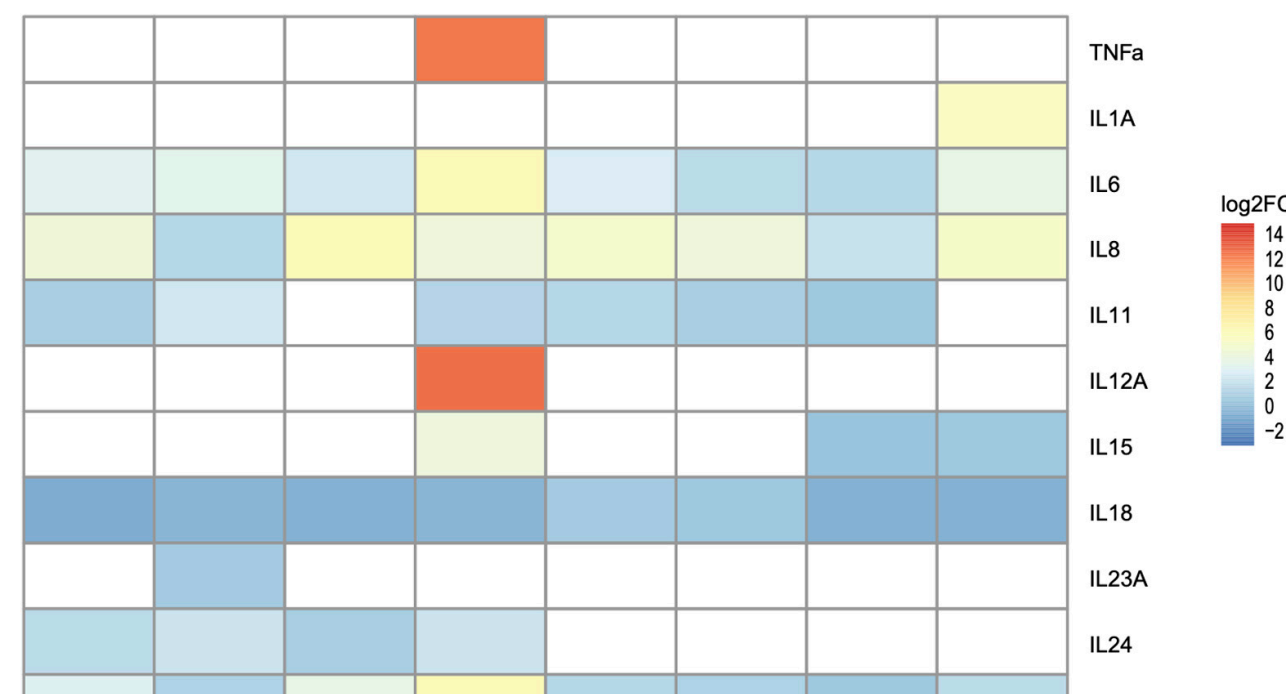

CCL2

CCL4

CCL5

CCL8

CCL17

CXCL1

CXCL2

CXCL3

CXCL6

CXCL12

CXCL14

CXCL16

CX3CL1

TNFSF7

TNFSF9

TNFSF10

TNFSF11

TNFSF12

TNFSF15

TNFSF18

HeV-6 hpi CedV-6 hpi HeV-24 hpi CedV-24 hpi HeV-6 hpi CedV-6 hpi HeV-24 hpi CedV-24 hpi

PaKi

HeLa

FIGURE 4 | Cytokine expression in HeV- or CedV-infected HeLa and PaKi cells at 6 or 24 hpi. The color scale for log2FC is shown at the top right of the figure. Genes that were not differentially expressed are colored white. HeV-6 hpi and CedV-6 hpi represent HeV- or CedV-infected corresponding PaKi or HeLa at 6 hpi. HeV-24 hpi and CedV-24 hpi represent HeV- or CedV-infected corresponding PaKi or HeLa at 24 hpi. 


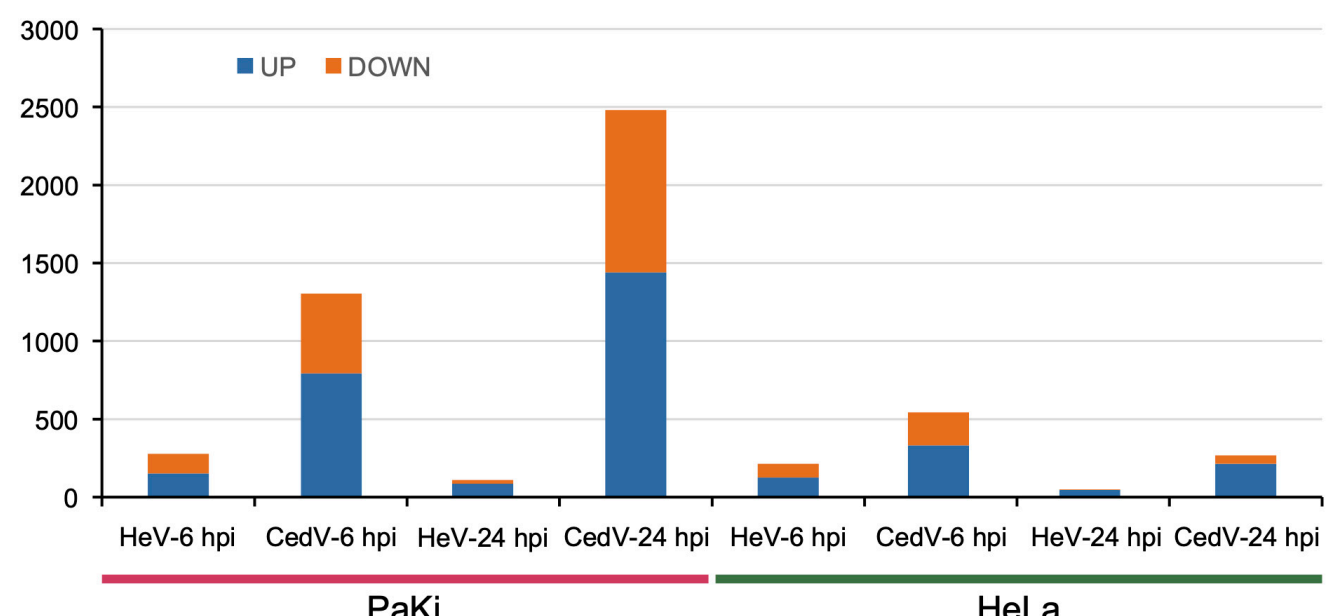

PaKi

HeLa

FIGURE 5 | Genes highly up- and downregulated (>2-fold change in expression as compared to uninfected cells) in HeV- or CedV-infected HeLa and PaKi cells at 6 or 24 hpi. HeV-6 hpi and CedV-6 hpi represent HeV- or CedV-infected corresponding PaKi or HeLa at 6 hpi. HeV-24 hpi and CedV-24 hpi represent HeV- or CedV-infected corresponding PaKi or HeLa at $24 \mathrm{hpi}$.

than did $\mathrm{HeV}$. It is possible that differences in the phosphoprotein gene coding strategy between the two viruses lead to host transcriptomic divergence and alterations in viral lethality. Further work is required to understand how henipaviruses evade the host immune response.

\section{MATERIALS AND METHODS}

\section{Cell Culture}

The $P$. alecto immortalized kidney-derived PaKi cell line (Crameri et al., 2009) and the human HeLa cell line were used in this study. Cell lines were grown in either Dulbecco's modified Eagle's medium (DMEM)/F12 (PaKiT03) or DMEM GlutaMAX (HeLa), both supplemented with $10 \%(\mathrm{v} / \mathrm{v})$ fetal calf serum (FCS) and $100 \mathrm{U} / \mathrm{mL}$ penicillin/streptomycin. All cells were incubated at $37^{\circ} \mathrm{C}$ in a humidified atmosphere containing $5 \% \mathrm{CO}_{2}$.

\section{HeV and CedV Infection of Bat and Human Cell Lines}

All virology work was conducted at the BSL-4 facility of the CSIRO Australian Animal Health Laboratory (Geelong, Australia). Approximately $2 \times 10^{7} \mathrm{PaKi}$ and $\mathrm{HeLa}$ cells per challenge were mock infected or infected with $\mathrm{HeV}$ (Hendra virus/Australia/horse/1994/Hendra) or CedV (Cedar virus/Australia/bat/2009/Cedar) for 6 or $24 \mathrm{~h}$ at a MOI of 10 . We performed three biological replicates for each challenge in $\mathrm{T} 75 \mathrm{~cm}^{2}$ flasks. All cells were harvested with trypsinization and resuspended in RLT buffer (Qiagen, Hilden, Germany). Mockinfected cells were used as controls.

\section{RNA Isolation, Sequencing, and RNA-Seq Analysis}

Total RNA was isolated using the Qiagen RNeasy Mini kit (Qiagen, Hilden, Germany) and treated with DNase I (Qiagen,
Hilden, Germany). The quality and quantity of total RNA was assessed using a Bioanalyzer (Agilent, Santa Carla, CA, United States). mRNA was sequenced on an Illumina HiSeq 2000 sequencer (Illumina, San Diego, CA, United States). Adapter sequences were trimmed from the resulting reads. The quality of reads was assessed using FastQC ${ }^{1}$. High-quality reads were mapped to either the $P$. alecto or human genomes using TopHat (version 2.1.1) (Trapnell et al., 2009). Based on the resulting alignments, we assembled our transcripts separately by challenge group. Grouped transcripts were assembled and merged using Cufflinks and Cuffmerge (version 2.2.1) (Trapnell et al., 2012). Differential expression analysis was performed with Cuffdiff (version 2.2.1) (Trapnell et al., 2012). Transcripts with a fragments per kilobase of transcript per million mapped reads (FPKM) value $<1$ before or after $\mathrm{HeV}$ or CedV infection were discarded. We considered a transcript statistically significant if the FDR-adjusted $p$-value of the test statistic ( $q$-value) was $<0.05$.

\section{Analysis of IFN Expression}

As IFN genes are only partially characterized in the P. alecto genome, PaKi RNA-seq reads were mapped separately to the $P$. alecto type I IFN locus (Zhou et al., 2016), IFN- $\lambda 1$ (GenBank accession no. HQ201956.1) and IFN- $\lambda 2$ (GenBank accession no. HQ201955.1) using bowtie2 (Langmead and Salzberg, 2012). We used these mappings to measure the expression of type I and III IFNs. We used SAMtools to compile and count the number of reads mapped (Li et al., 2009).

\section{Functional Enrichment Analysis}

To predict the molecular interactions of the DEGs, we performed KEGG enrichment analysis with KOBAS (Xie et al., 2011), using a hypergeometric test. We considered KEGG pathways with $p<0.05$ significantly enriched. The $-\log 10$ ( $p$-value) indicates

\footnotetext{
${ }^{1}$ http://www.bioinformatics.babraham.ac.uk/projects/fastqc/
} 
the enrichment score, which represents the significance of the corresponding pathway enrichment.

\section{DATA AVAILABILITY STATEMENT}

The original contributions presented in the study are publicly available. This data can be found here: https://bigd.big.ac.cn/gsa, accession number CRA002578.

\section{AUTHOR CONTRIBUTIONS}

L-FW and JC designed the research and supervised the experiments. JC, MT, and GM performed the experiments. JC and MC analyzed the data. JC, MC, and L-FW wrote the manuscript.

\section{REFERENCES}

Baker, M. L., Schountz, T., and Wang, L. F. (2013). Antiviral immune responses of bats: a review. Zoonoses Public Health 60, 104-116. doi: 10.1111/j.1863-2378. 2012.01528.x

Barber, G. N. (2001). Host defense, viruses and apoptosis. Cell Death Differ. 8, 113-126. doi: 10.1038/sj.cdd.4400823

Crameri, G., Todd, S., Grimley, S., McEachern, J. A., Marsh, G. A., Smith, C., et al. (2009). Establishment, immortalisation and characterisation of pteropid bat cell lines. PLoS One 4:e8266. doi: 10.1371/journal.pone.0008266

Cuda, C. M., Pope, R. M., and Perlman, H. (2016). The inflammatory role of phagocyte apoptotic pathways in rheumatic diseases. Nat. Rev. Rheumatol. 12, 543-558. doi: 10.1038/nrrheum.2016.132

Eaton, B. T., Broder, C. C., Middleton, D., and Wang, L. F. (2006). Hendra and Nipah viruses: different and dangerous. Nat. Rev. Microbiol. 4, 23-35. doi: $10.1038 /$ nrmicro 1323

Fensterl, V., and Sen, G. C. (2015). Interferon-induced Ifit proteins: their role in viral pathogenesis. J. Virol. 89, 2462-2468. doi: 10.1128/jvi.02744- 14

Field, H., Young, P., Yob, J. M., Mills, J., Hall, L., and Mackenzie, J. (2001). The natural history of Hendra and Nipah viruses. Microbes Infect. 3, 307-314. doi: 10.1016/s1286-4579(01)01384-3

Field, H. E. (2016). Hendra virus ecology and transmission. Curr. Opin. Virol. 16, 120-125. doi: 10.1016/j.coviro.2016.02.004

Glennon, N. B., Jabado, O., Lo, M. K., and Shaw, M. L. (2015). Transcriptome profiling of the virus-induced innate immune response in Pteropus vampyrus and its attenuation by Nipah virus interferon antagonist functions. J. Virol. 89, 7550-7566. doi: 10.1128/JVI.00302-15

Khandia, R., Singhal, S., Kumar, U., Ansari, A., Tiwari, R., Dhama, K., et al. (2019). Analysis of Nipah virus codon usage and adaptation to hosts. Front. Microbiol. 10:886. doi: 10.3389/fmicb.2019.00886

Klotman, M. E., and Chang, T. L. (2006). Defensins in innate antiviral immunity. Nat. Rev. Immunol. 6, 447-456. doi: 10.1038/nri1860

Langmead, B., and Salzberg, S. L. (2012). Fast gapped-read alignment with Bowtie 2. Nat. Methods 9, 357-359. doi: 10.1038/nmeth.1923

Li, H., Handsaker, B., Wysoker, A., Fennell, T., Ruan, J., Homer, N., et al. (2009). The sequence alignment/map format and SAMtools. Bioinformatics 25, 20782079. doi: 10.1093/bioinformatics/btp352

Marsh, G. A., de Jong, C., Barr, J. A., Tachedjian, M., Smith, C., Middleton, D., et al. (2012). Cedar virus: a novel Henipavirus isolated from Australian bats. PLoS Pathog. 8:e1002836. doi: 10.1371/journal.ppat.1002836

Rahman, M. M., and McFadden, G. (2011). Modulation of NF-kappaB signalling by microbial pathogens. Nat. Rev. Microbiol. 9, 291-306. doi: 10.1038/ nrmicro2539

Ranjan, P., Singh, N., Kumar, A., Neerincx, A., Kremmer, E., Cao, W., et al. (2015). NLRC5 interacts with RIG-I to induce a robust antiviral response against influenza virus infection. Eur. J. Immunol. 45, 758-772. doi: 10.1002/ eji.201344412

Sadler, A. J., and Williams, B. R. (2008). Interferon-inducible antiviral effectors. Nat. Rev. Immunol. 8, 559-568. doi: 10.1038/nri2314

\section{FUNDING}

This study was supported by National Science and Technology Major Project (Grant No. 2018ZX10301101), CAS Pioneer Hundred Talents Program to JC; Singapore National Research Foundation (Grant No. NRF2012NRF-CRP001-056), and CSIRO OCE Science Leader award to L-FW.

\section{SUPPLEMENTARY MATERIAL}

The Supplementary Material for this article can be found online at: https://www.frontiersin.org/articles/10.3389/fmicb. 2020.00986/full\#supplementary-material

Satoh, T., Kato, H., Kumagai, Y., Yoneyama, M., Sato, S., Matsushita, K., et al. (2010). LGP2 is a positive regulator of RIG-I- and MDA5-mediated antiviral responses. Proc. Natl. Acad. Sci. U.S.A. 107, 1512-1517. doi: 10.1073/pnas. 0912986107

Satterfield, B. A., Cross, R. W., Fenton, K. A., Agans, K. N., Basler, C. F., Geisbert, T. W., et al. (2015). The immunomodulating V and $\mathrm{W}$ proteins of Nipah virus determine disease course. Nat. Commun. 6:7483. doi: 10.1038/ncomm s8483

Schmid, S., Mordstein, M., Kochs, G., García-Sastre, A., and Tenoever, B. R. (2010). Transcription factor redundancy ensures induction of the antiviral state. J. Biol. Chem. 285, 42013-42022. doi: 10.1074/jbc.M110.165936

Shuai, K., and Liu, B. (2003). Regulation of JAK-STAT signalling in the immune system. Nat. Rev. Immunol. 3, 900-911. doi: 10.1038/nri1226

Trapnell, C., Pachter, L., and Salzberg, S. L. (2009). TopHat: discovering splice junctions with RNA-Seq. Bioinformatics 25, 1105-1111. doi: 10.1093/ bioinformatics/btp120

Trapnell, C., Roberts, A., Goff, L., Pertea, G., Kim, D., Kelley, D. R., et al. (2012). Differential gene and transcript expression analysis of RNA-seq experiments with TopHat and Cufflinks. Nat. Protoc. 7, 562-578. doi: 10.1038/nprot.2012. 016

Wynne, J. W., Shiell, B. J., Marsh, G. A., Boyd, V., Harper, J. A., Heesom, K., et al. (2014). Proteomics informed by transcriptomics reveals Hendra virus sensitizes bat cells to TRAIL-mediated apoptosis. Genome Biol. 15:532. doi: 10.1186/PREACCEPT-1718798964145132

Xie, C., Mao, X., Huang, J., Ding, Y., Wu, J., Dong, S., et al. (2011). KOBAS 2.0: a web server for annotation and identification of enriched pathways and diseases. Nucleic Acids Res. 39, W316-W322. doi: 10.1093/nar/gkr483

Zhang, G., Cowled, C., Shi, Z., Huang, Z., Bishop-Lilly, K. A., Fang, X., et al. (2013). Comparative analysis of bat genomes provides insight into the evolution of flight and immunity. Science 339, 456-460. doi: 10.1126/science.1230835

Zhou, P., Cowled, C., Todd, S., Crameri, G., Virtue, E. R., Marsh, G. A., et al. (2011). Type III IFNs in pteropid bats: differential expression patterns provide evidence for distinct roles in antiviral immunity. J. Immunol. 186, 3138-3147. doi: 10.4049/jimmunol.1003115

Zhou, P., Tachedjian, M., Wynne, J. W., Boyd, V., Cui, J., Smith, I., et al. (2016). Contraction of the type I IFN locus and unusual constitutive expression of IFNalpha in bats. Proc. Natl. Acad. Sci. U.S.A. 113, 2696-2701. doi: 10.1073/pnas. 1518240113

Conflict of Interest: The authors declare that the research was conducted in the absence of any commercial or financial relationships that could be construed as a potential conflict of interest.

Copyright (c) 2020 Chen, Tachedjian, Marsh, Cui and Wang. This is an open-access article distributed under the terms of the Creative Commons Attribution License (CC BY). The use, distribution or reproduction in other forums is permitted, provided the original author(s) and the copyright owner(s) are credited and that the original publication in this journal is cited, in accordance with accepted academic practice. No use, distribution or reproduction is permitted which does not comply with these terms. 